\title{
Determinación del rendimiento de un motor de Stirling usando Arduino: una propuesta para la enseñanza de la termodinámica en los cursos introductorios
}

Determining the efficiency of a Stirling engine using Arduino: a proposal for teaching thermodynamics in introductory courses

\author{
F. Savall-Alemany ${ }^{* 1,2}$, M. Esparza-García ${ }^{3}$, J. F. Álvarez-Herrero ${ }^{1}$, S. Rosa-Cintas ${ }^{1,4}$ \\ ${ }^{1}$ Universidad de Alicante, Facultad de Educación, Departamento de Didáctica \\ General y Didácticas Específicas, Alicante, España. \\ ${ }^{2}$ IES Veles e Vents, Gandia, València, España. \\ ${ }^{3}$ IES Ausiàs March, Gandia, València, España. \\ ${ }^{4}$ Universidad de Alicante, Instituto Universitario de Física Aplicada a la Ciencia y la Tecnología, España.
}

Recibida en 07 de Julio, 2020. Revisado en 28 de Agosto, 2020. Aceptado en 07 de octubre, 2020.

\begin{abstract}
Durante los últimos años la investigación en didáctica de la Física ha destacado el interés que tiene la plataforma Arduino y los sensores asociados a ella en la mejora de la enseñanza. En este trabajo presentamos una propuesta para el estudio de la termodinámica en los cursos introductorios. Hemos construido un motor de Stirling casero con materiales de bajo coste. A partir de un estudio teórico del ciclo termodinámico asociado al motor, proponemos diversas estrategias para medir su rendimiento. A continuación, describimos el montaje experimental, basado en sensores Arduino, que nos permite registrar las medidas necesarias para desarrollar las estrategias propuestas. Los resultados obtenidos ponen de manifiesto el bajo rendimiento de los motores térmicos y las diferencias existentes entre los ciclos teóricos y los experimentales.
\end{abstract}

Palabras clave: Motor térmico, motor de Stirling, termodinámica, Arduino.

\begin{abstract}
During the last few years, research in physics education has highlighted the interest of the Arduino platform and its associated sensors in improving teaching. In this paper we present a proposal for the study of thermodynamics in introductory courses. We have built a home-made Stirling engine with low-cost materials. From a theoretical study of the thermodynamic cycle associated to the engine, we propose several strategies to measure its efficiency. Next, we describe the experimental setup, based on Arduino sensors, that allows us to record the necessary measurements to develop the proposed strategies. The results obtained show the low efficiency of thermal engines and the differences between the theoretical and experimental thermodynamic cycles.
\end{abstract}

Keywords: Heat engine, Stirling engine, thermodynamics, Arduino.

\section{Introducción}

La plataforma Arduino se ha revelado, durante los últimos años, como un instrumento especialmente útil en la mejora de la enseñanza de la Física. Permite llevar a cabo investigaciones experimentales en el aula, integrando diversas disciplinas, con un coste económico asumible por escuelas de secundaria y universidades [1, 2]. Así mismo, se ha constatado que a través de la implicación de los estudiantes en actividades experimentales desarrolladas con Arduino se produce una mejora en el interés de los estudiantes y en el aprendizaje [3, 4]. La introducción de Arduino ha sido amplia en lo que a niveles educativos y a campos de la física y la ingeniería se refiere. En la literatura didáctica se pueden

\footnotetext{
${ }^{*}$ Correo electrónico: paco.savall@ua.es
}

encontrar propuestas orientadas tanto a la escuela secundaria como a la enseñanza universitaria relacionadas con la medida de la tensión superficial [5], la velocidad del sonido 6] o la constante de Planck [7], el estudio de la caída libre [8], la radiación solar [9] o el funcionamiento de placas fotovoltaicas [10, por poner solo algunos ejemplos.

Los trabajos relacionados con las propiedades de los gases son, posiblemente, los que más han aprovechado esta nueva tecnología. Se ha descrito su uso, entre otros, en el estudio de la relación entre las variables de estado de un gas [11, en la puesta a prueba de la ley de Avogadro para los gases [3], la determinación del cero absoluto a partir de las propiedades del gas ideal [12] o la variación de presión en un tubo de Venturi [13. En todos estos trabajos se pone de manifiesto que los sensores de temperatura y presión que se pueden usar 
conjuntamente con la placa Arduino permiten tomar gran cantidad de medidas en intervalos breves de tiempo y que su reducido tamaño permite introducirlos en recipientes y dispositivos en los que no se pueden introducir termómetros o barómetros más tradicionales.

El objetivo de nuestro trabajo es determinar el rendimiento de un modelo escolar de motor de Stirling. Desde el punto de vista didáctico, el interés del motor de Stirling para el estudio de los ciclos termodinámicos y los motores en el aula se ha evidenciado en diversas publicaciones a lo largo del tiempo [14-16]. Más recientemente, el uso de sensores electrónicos y ordenadores ha permitido profundizar en su estudio [17, 18. Desde el punto de vista curricular, la relevancia tecnológica, científica y social que tienen los motores térmicos ha llevado a que se contemple su estudio en los currículums de la enseñanza secundaria 19. Para nuestra investigación, a diferencia de la mayoría de las anteriores, hemos decidido usar un motor de Stirling construido por nosotros mismos en lugar de un motor comercial en tanto que nos permite una mayor manipulación. Así mismo, hemos usado sensores económicos y la placa Arduino UNO por su bajo coste y facilidad de uso. Hemos construido el motor de Stirling empleando materiales comunes y económicos, siguiendo los pasos que detallamos en la sección 2 Un estudio teórico del ciclo termodinámico del motor (sección 3) nos ha permitido establecer diversas estrategias experimentales para determinar su rendimiento. Las presentamos y analizamos en la sección 4 En la sección 5 explicamos el montaje llevado a cabo para la recogida de datos. Finalmente, presentamos y analizamos los resultados obtenidos, comparando cada una de las estrategias usadas para determinar el rendimiento del motor (secciones 6 y 7 ).

\section{Montaje del Motor de Stirling}

En el presente trabajo, hemos construido el motor de Stirling siguiendo las indicaciones de Boada [20]. Para ello, hemos realizado las siguientes tareas:

1. Disponemos una jeringuilla de vidrio sujeta con dos bridas a un tornillo vertical, incrustado a su vez en una tabla de madera. Posteriormente, enganchamos a la jeringuilla una placa metálica debajo de la cual situamos un sensor de posición que nos permitirá obtener medidas de la posición de la placa (Figura 19).

2. Dos placas metálicas con forma de L, fijadas a la tabla de madera, sirven de soporte para un tubo de ensayo, que se posiciona horizontalmente. El tubo de ensayo lleva adaptado, aproximadamente en el centro, un eje metálico horizontal perpendicular al tubo sobre el cual puede bascular (Figura 1 1 ).

3. En el interior del tubo introducimos dos canicas (esferas de vidrio) con un diámetro ligeramente inferior al del tubo. Para evitar impactos entre las esferas y el fondo del tubo de vidrio, que puedan fracturarlo, lo protegemos con un trozo de lana metálica (Figura 1;).

4. Tapamos el tubo con un tapón de goma de dos orificios (de los que se usan normalmente en los laboratorios de química). A través de uno de los orificios, pasamos un tubo de vidrio que, mediante un pequeño tubo de goma, conecta el interior del tubo de ensayo con el interior de la jeringuilla, formando un único compartimento estanco (no puede salir ni entrar aire, sólo puede circular del tubo de ensayo a la jeringuilla).

5. Utilizamos el segundo de los orificios del tapón para introducir un sensor electrónico de presión y temperatura, que situaremos tan cerca del tapón como sea posible. Sellamos el orificio con cola térmica para evitar fugas de aire a través de él. Para evitar daños sobre el sensor, hemos dispuesto un tornillo que hace de tope y evita el impacto de las esferas de vidrio sobre el mismo (Figura 1 1 ).

6. Tanto el sensor de posición como el sensor de presión y temperatura se conectan a una placa Arduino UNO y esta se conecta, a su vez, a un ordenador. Un mechero de alcohol aportará la energía necesaria para hacer funcionar el motor. En la Figura 1, se puede ver el montaje completo del motor.

\section{Fundamentos Físicos del Motor de Stirling}

Los motores térmicos son máquinas en las cuales un gas sigue un ciclo de calentamiento y enfriamiento que le lleva a realizar ciclos de expansión y compresión durante los cuales realiza un trabajo. De forma general y esquemática, los intercambios de energía que tienen lugar entre un motor y su entorno se pueden representar mediante el diagrama de la Figura 2. El gas que acciona el motor absorbe calor $\left(\mathrm{Q}_{\mathrm{c}}\right)$ de un foco caliente. Parte de esta energía la convierte en trabajo útil (W) y otra parte la transfiere en forma de calor a un foco frío $\left(\mathrm{Q}_{\mathrm{f}}\right)$.

Un motor con alto rendimiento es aquel que puede hacer una gran cantidad de trabajo. En virtud del principio de conservación de la energía, el trabajo realizado por el motor no puede ser superior al calor absorbido del foco caliente. Es por ello que el rendimiento $(\varepsilon)$ se determina como la relación entre el trabajo hecho por el motor y el calor absorbido del foco caliente:

$$
\varepsilon=\frac{W}{Q_{\text {absorbido }}}
$$

El motor de Stirling ideal consta de 4 etapas que se distinguen claramente en su ciclo termodinámico (Figura 3).

La primera etapa (de 11 a 2 consiste en un calentamiento a volumen constante. El calor absorbido 

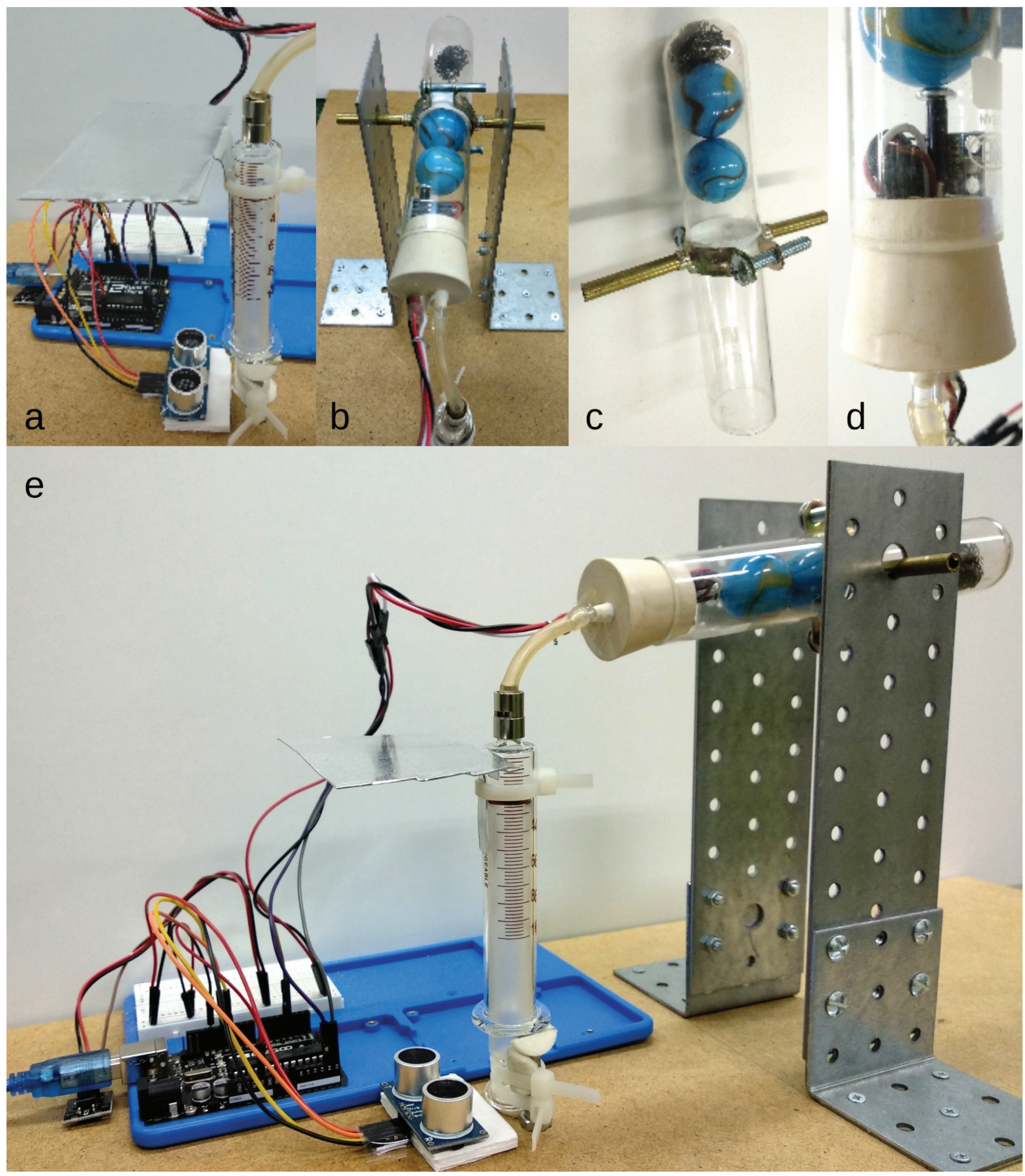

Figura 1: En las imágenes se presentan detalles del montaje del motor. La imagen 1a muestra la disposición del sensor de posición y la placa metálica que permite medir la posición de la jeringuilla. La imagen 1b muestra la disposición del tubo de ensayo y los elementos de su interior. En 1c se observa el eje con más detalle. La imagen 1d muestra cómo se ha situado el sensor de presión y temperatura y cómo se ha protegido. La imagen 1e muestra el montaje completo. 


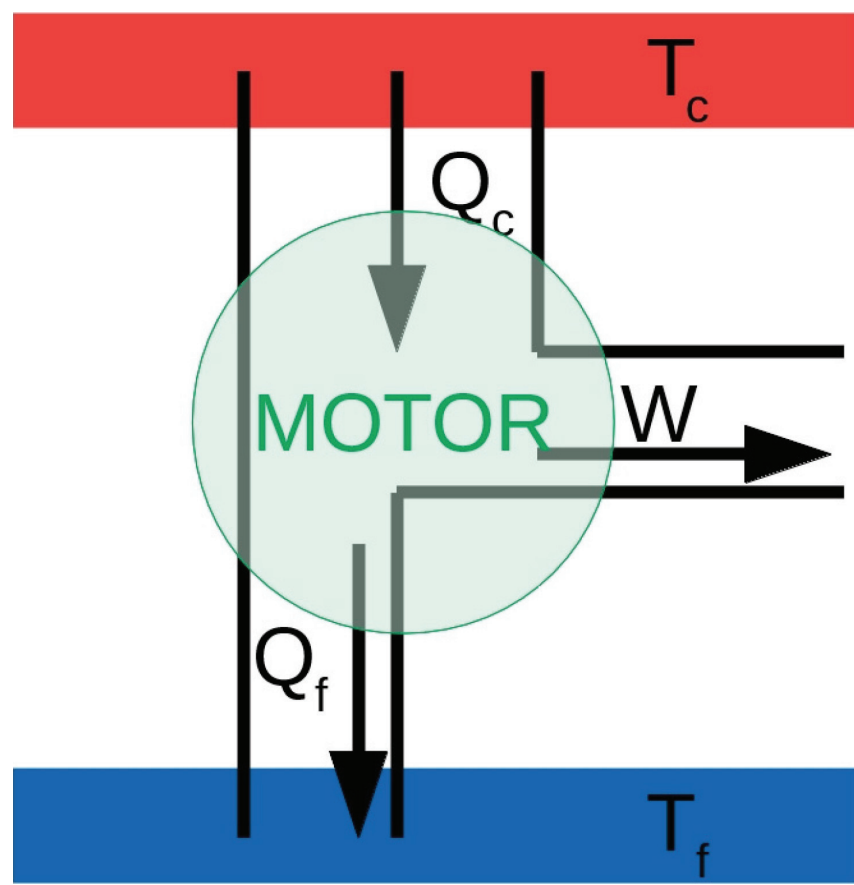

Figura 2: Representación esquemática del funcionamiento general de un motor térmico. El motor absorbe calor de un foco caliente $\left(Q_{c}\right)$. Convierte parte de la energía recibida en trabajo (W) y transfiere la energía restante a un foco frío $\left(Q_{\mathrm{f}}\right)$.

durante la etapa es:

$$
Q_{12}=n c_{v}\left(T_{c}-T_{f}\right)
$$

La segunda etapa (de 2 a 3) consiste en una expansión isoterma. Durante esta etapa el gas (considerado ideal) no experimenta variación de energía interna, por lo cual el calor absorbido debe ser igual al trabajo realizado:

$$
\begin{aligned}
Q_{23} & =W_{23}=\int_{V_{1}}^{V_{2}} p \cdot d V=\int_{V_{1}}^{V_{2}} \frac{n R T_{c}}{V} \cdot d V \\
& =n R T_{c} \ln \frac{V_{2}}{V_{1}}
\end{aligned}
$$

La tercera etapa (de 3 a 4 consiste en un enfriamiento a volumen constante. El trabajo realizado por el gas es nulo, por lo que disminuye su energía interna desprendiendo calor de acuerdo con la expresión:

$$
Q_{34}=n c_{v}\left(T_{f}-T_{c}\right)
$$

La cuarta etapa (de 4 a 1) consiste en una compresión a temperatura constante. De manera similar a lo ocurrido en la segunda etapa:

$$
\begin{aligned}
Q_{41} & =W_{41}=\int_{V_{2}}^{V_{1}} p \cdot d V=\int_{V_{2}}^{V_{1}} \frac{n R T_{f}}{V} \cdot d V \\
& =n R T_{f} \ln \frac{V_{1}}{V_{2}}=-n R T_{f} \ln \frac{V_{2}}{V_{1}}
\end{aligned}
$$

Los motores de Stirling suelen disponer de un regenerador que absorbe parte del calor que desprende el gas al pasar del foco caliente al foco frío y lo vuelve a aportar en el foco caliente durante el siguiente ciclo. Con ello se consigue disminuir el calor aportado al motor, aumentando su rendimiento. Dado que nuestro motor no dispone de regenerador, hemos decidido no desarrollar este aspecto.

Nuestro motor también funciona mediante ciclos de calentamiento y enfriamiento del gas que contiene. El

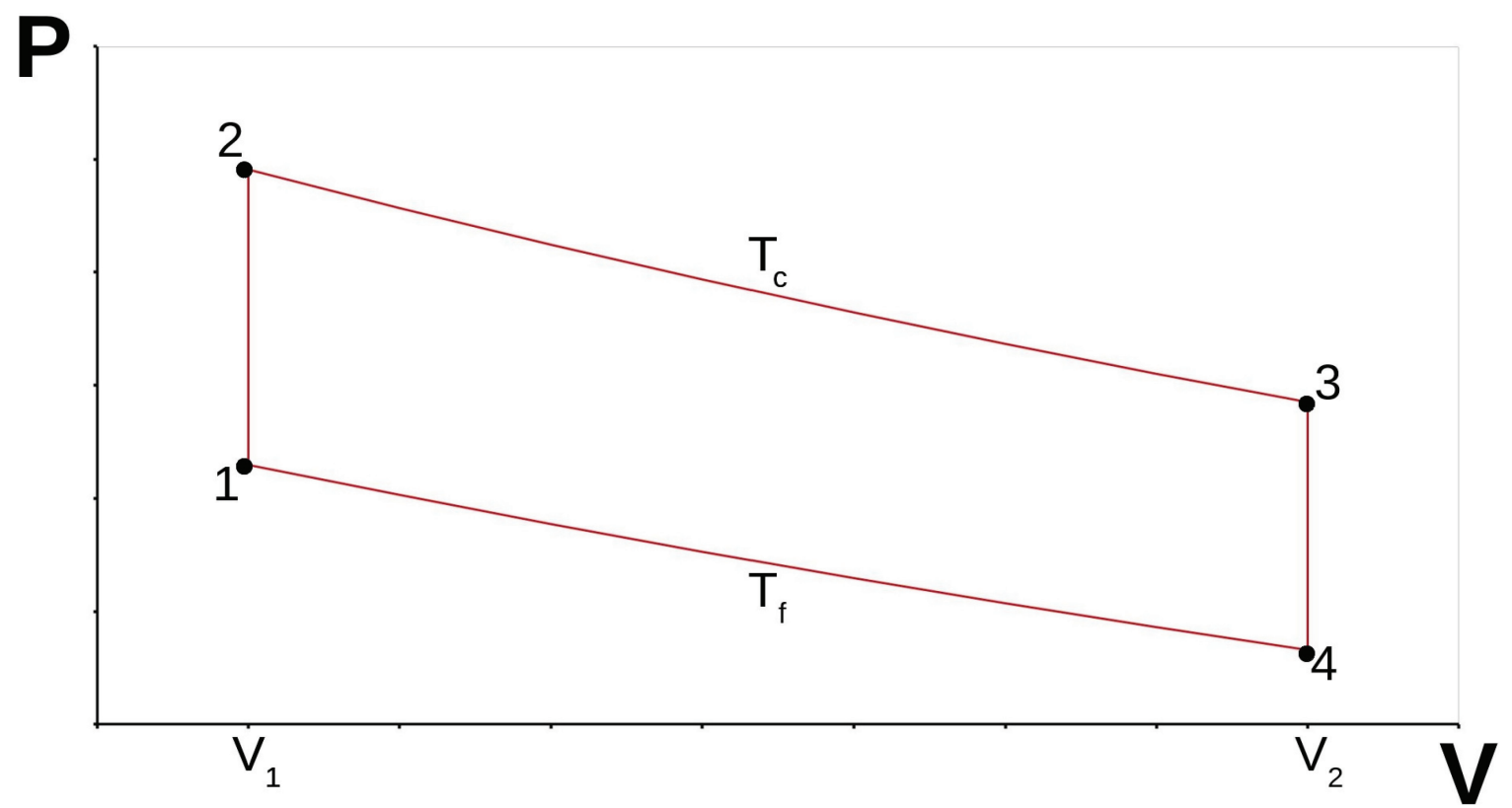

Figura 3: Ciclo termodinámico de un motor de Stirling ideal. Se distinguen 4 etapas. Un calentamiento isócoro (1 a 2), una expansión isoterma (2 a 3), un enfriamiento isócoro (3 a 4) y una compresión isoterma (4 a 1). 


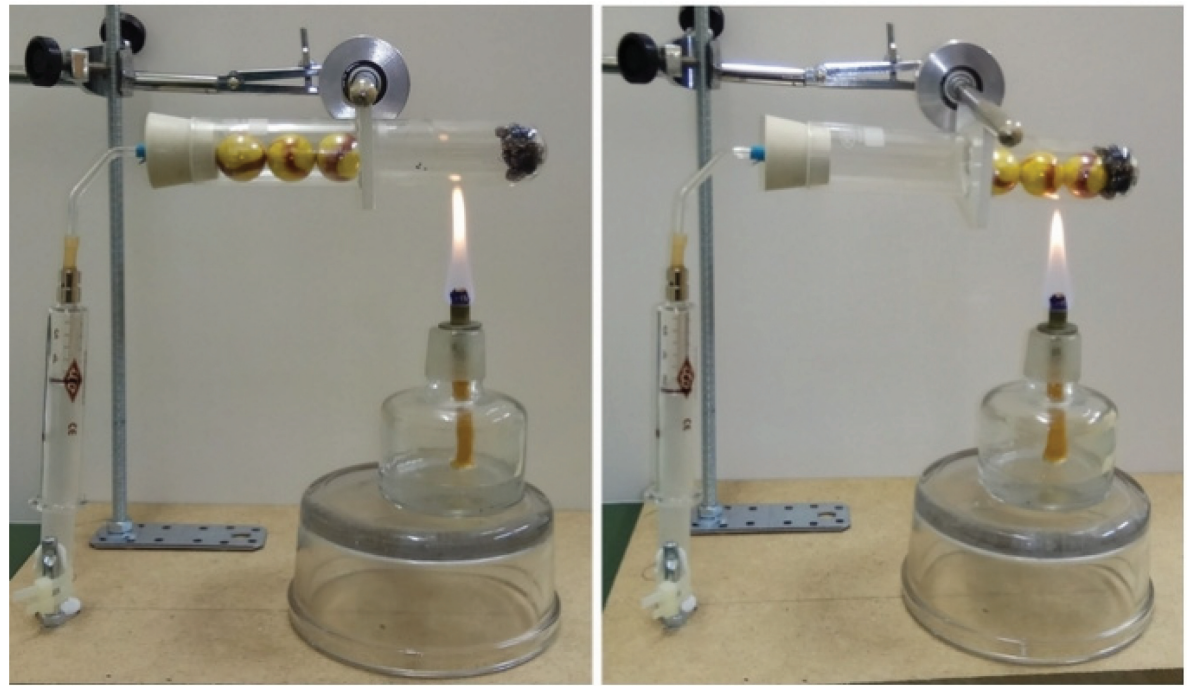

Figura 4: Versión simplificada del motor de Stirling usado durante la investigación. El foco caliente corresponde a la parte que se encuentra justo sobre la llama mientras que el foco frío corresponde a la parte del tubo de ensayo más próxima al tapón. La imagen izquierda se corresponde con el inicio de la primera etapa, donde el gas del interior del tubo de ensayo es calentado por el mechero de alcohol. La imagen derecha muestra el momento en el que la expansión del gas (segunda etapa) produce la elevación de la jeringuilla y el desplazamiento de las canicas al fondo del tubo, lo que permitirá el enfriamiento del gas en la zona fría del sistema (tercera etapa).

foco caliente se sitúa en la zona del tubo de ensayo calentada por la llama, mientras que el foco frío se encuentra en el extremo opuesto, en la zona del tubo de ensayo próxima al tapón de goma. Inicialmente, el dispositivo se encuentra en la posición mostrada en la parte izquierda de la Figura 4

Durante una primera etapa, se calienta el extremo del tubo de ensayo, aumentando la temperatura del gas que contiene. El aumento de presión que se produce con el incremento progresivo de la temperatura lleva a la segunda etapa, en la cual se produce una expansión del gas. La jeringuilla, que es la única parte del motor que puede variar su volumen, asciende. Cuando el desplazamiento es suficientemente grande, el tubo de ensayo se inclina hacia el extremo opuesto (mostrado en la imagen derecha de la Figura 4 y las canicas caen hasta el fondo del tubo, desplazando hacia el tapón al aire que ocupaba dicho espacio. En la zona próxima al tapón la temperatura es menor, de manera que en la tercera etapa el aire desplazado desde el extremo caliente se enfría al llegar a esta zona. El enfriamiento hace que la presión del sistema disminuya. Así pues, durante la cuarta etapa la jeringuilla baja, recupera su volumen inicial e inclina de nuevo el tubo de ensayo hacia la izquierda (como se muestra en la imagen izquierda de la Figura 4). Como consecuencia, las canicas se mueven de nuevo hacia el tapón y desplazan el aire que allí se encuentra hacia el extremo caliente del tubo, reiniciando el ciclo del motor.

La observación del movimiento del motor nos permite deducir, a priori, que la primera etapa se corresponde con un calentamiento isócoro, en tanto que no hay movimiento durante un breve período de tiempo.
Así mismo, la tercera etapa se corresponde con un enfriamiento isócoro. Sin embargo, no se puede afirmar que la segunda y cuarta etapa se correspondan con procesos isotermos. En la sección de resultados discutiremos en qué medida el ciclo termodinámico de nuestro motor se corresponde con un ciclo de Stirling ideal.

\section{Procedimientos Seguidos para Medir el Rendimiento del Motor}

A pesar de la aparente sencillez de la ecuación 1 1 son diversos los procedimientos que se pueden seguir para determinar el rendimiento del motor. Detallamos a continuación aquellos que hemos puesto en práctica.

\subsection{Determinación del rendimiento a partir del trabajo experimental del motor}

Se podría conectar al motor un dispositivo que permita elevar un objeto de masa conocida, de manera que el trabajo realizado por el motor se determinaría a partir de la variación de energía potencial del objeto que asciende. Se podría medir, al mismo tiempo, la cantidad de combustible que consume el mechero y determinar la energía que aporta dicha cantidad de combustible. Relacionando ambas magnitudes podríamos obtener el rendimiento experimental del motor.

En nuestro caso, debido a la fragilidad de los elementos de vidrio usados para construir el motor, no consideramos viable la posibilidad de conectar un dispositivo que permita elevar objetos. Por ello, hemos medido experimentalmente el trabajo realizado por el gas (W) a 
lo largo de un ciclo usando la ecuación:

$$
W=p \cdot \Delta V
$$

El sensor de presión introducido dentro del tubo de ensayo nos proporciona medidas de la presión del gas, mientras que el sensor de posición nos da información sobre la posición de la jeringuilla, lo que nos permite calcular la variación del volumen que experimenta el sistema, aspecto que requiere una calibración previa.

Hemos calibrado el sensor de posición para que aporte medidas de volumen, determinando la recta de regresión que relaciona la posición de la placa unida a la jeringuilla y el volumen de aire contenido en la jeringuilla. Para ello, manualmente hemos desplazado la jeringuilla hasta la posición de $2 \mathrm{ml}$ y hemos anotado la posición de la placa que indica el sensor de posición. A continuación, hemos desplazado la jeringuilla hasta la posición de $3 \mathrm{ml}$ y hemos anotado de nuevo la posición de la placa metálica que indica el sensor. Hemos repetido las medidas para todos los volúmenes indicados en la escala de la jeringuilla. Con los resultados obtenidos hemos determinado la correspondiente recta de regresión que relaciona la posición de la placa con el volumen de la jeringuilla. La ecuación obtenida la hemos incorporado al programa ejecutado por Arduino para obtener directamente medidas de volumen.

La placa Arduino, una vez programada (ver sección 5 y el Anexo del artículo) aportará las medidas de volumen y presión necesarias para determinar el trabajo realizado por el gas. En la sección de resultados se presentan las medidas y los cálculos realizados a partir de ellas.

Así mismo, hemos determinado el calor aportado al motor a partir de la cantidad de alcohol consumida en el mechero. El combustible usado es alcohol $96^{\circ}$ comercial (disolución de etanol con un $96 \%$ de riqueza en volumen). Hemos medido la cantidad de alcohol que consume el mechero en 2 minutos y, a partir de la entalpía de combustión tabulada para el etanol, hemos determinado la energía que proporciona por unidad de tiempo. Hay que destacar que este procedimiento no permite conocer exactamente la cantidad de calor absorbida por el motor, en tanto que la llama puede situarse en diversas posiciones respecto al motor. Así mismo, nuestro motor no dispone de un sistema específico de captación de calor. El calor captado por el motor será, con todo, una fracción de la energía producida en la combustión del alcohol. Este procedimiento, por tanto, sobredimensiona el valor de $\mathrm{Q}_{\mathrm{ab}}$. Valoraremos su impacto sobre el cálculo del rendimiento del motor en la sección de resultados.

\subsection{Determinación del rendimiento a partir del ciclo termodinámico ideal}

Conociendo el ciclo termodinámico del motor podemos determinar el calor absorbido por el gas y el trabajo realizado por el mismo. El rendimiento del motor de Stirling ideal sin regenerador, de acuerdo con las ecuaciones 2 a 5. se calcula como:

$$
\begin{aligned}
\varepsilon & =\frac{W}{Q_{a b}}=\frac{n R T_{c} \ln \frac{V_{2}}{V_{1}}-n R T_{f} \ln \frac{V e_{2}}{V_{1}}}{n R T_{c} \ln \frac{V_{2}}{V_{1}}+n c_{v}\left(T_{c}-T_{f}\right)} \\
& =\frac{R\left(T_{c}-T_{f}\right) \ln \frac{V_{2}}{V_{1}}}{R T_{c} \ln \frac{V_{2}}{V_{1}}+c_{v}\left(T_{c}-T_{f}\right)}
\end{aligned}
$$

Los valores $V_{1}$ y $V_{2}$ los hemos determinado a partir del volumen de las canicas, en tanto que son las canicas las que desplazan el aire que se somete al ciclo de calentamiento y enfriamiento. Hemos medido $T_{f}$ y la presión con el sensor de presión y temperatura situado junto al tapón. Usando los valores de $\mathrm{V}_{1}, \mathrm{~V}_{2}, \mathrm{~T}_{\mathrm{f}}$ y de la presión a lo largo del ciclo, y considerando que el aire del interior del motor se comporta como un gas ideal,

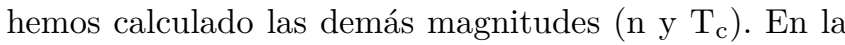
sección de resultados, usando los datos experimentales obtenidos, presentaremos más detalles sobre cómo hemos hecho estos cálculos.

\subsection{Determinación del rendimiento a partir de procedimientos mixtos}

Como hemos indicado, el ciclo que lleva a cabo el motor experimental puede no coincidir con el ciclo ideal. Así mismo, la dificultad experimental para medir determinadas magnitudes obliga a realizar estimaciones a partir del ciclo ideal. En la sección de resultados discutimos con más detalle estos aspectos, en base a las medidas realizadas en nuestro estudio.

\section{Montaje y Programación de los Sensores Electrónicos}

Hemos conectado los siguientes componentes a una placa Arduino $\mathrm{UNO}^{1}$ En la Tabla 1 se indica en qué pin se ha conectado cada uno de ellos:

- Sensor BMP180: Usado para medir la presión del gas y la temperatura del foco frío.

- Sensor HC-SR04: Usado para medir el volumen del gas en el interior de la jeringuilla, calibrado como hemos explicado anteriormente. Este sensor, debido a sus características técnicas, tiene una precisión de $\pm 3 \mathrm{~mm}$. Además, por su propio funcionamiento, puede fallar al llevar a cabo medidas en intervalos de tiempo muy breves. Todo ello obliga a un tratamiento detallado de las medidas registradas. Concretamente, como comentamos también en la sección de resultados, hemos filtrado las medidas registradas eliminando los registros que carecen de sentido físico y hemos realizado

\footnotetext{
1 Para una descripción detallada del funcionamiento, conexión y programación de cada componente, véase, por ejemplo, https://www.luisllamas.es/tutoriales-de-Arduino/
} 
Tabla 1: Se indica el pin de la placa Arduino UNO al que se conecta cada terminal de los sensores empleados.

\begin{tabular}{cccc}
\hline Pin Arduino & Sensor & Sensor & \\
UNO & BMP180 & HC-SR04 & Botón \\
\hline VCC & VCC & VCC & VCC \\
GND & GND & GND & GND \\
A4 & SCL & & \\
A5 & SDA & & \\
3 & & & S \\
11 & & ECHO & \\
12 & & TRIG & \\
\hline
\end{tabular}

medidas correspondientes a diversos ciclos del motor con el fin de obtener un promedio que minimizase el efecto de las fluctuaciones indeseadas.

- Botón: Hemos incorporado un botón que nos permite controlar el flujo de información que se transmite al ordenador.

El programa empleado, junto con los comentarios necesarios para su comprensión y manipulación, se presenta en el documento Anexo. A grandes rasgos, tiene la siguiente estructura: se empieza indicando las librerías que se usan y declarando las variables, a continuación se declaran e inician los sensores. Le sigue la función que se repite en bucle. En ella se lee el estado del botón y solo cuando está pulsado se lleva a cabo alguna acción. Con el botón pulsado el programa procede a la lectura de la posición de la placa metálica y a la determinación del volumen del aire en la jeringuilla. A continuación, mide la presión del gas y la temperatura del foco frío y finalmente transmite todas las medidas al ordenador a través del puerto usb.

El dispositivo realiza el máximo de medidas que le permite su velocidad de procesamiento. Junto con las medidas de volumen, presión y temperatura obtenemos el registro de tiempo que ofrece el monitor en serie de la IDE de Arduino. Todas las medidas se registran en el monitor en serie, ordenadas en filas y separadas por un punto y coma (;) para facilitar su traslado a una hoja de cálculo. Mediante una acción de copy-paste pasamos las medidas del monitor serial a una hoja de cálculo para su procesamiento. Aunque las medidas de presión, volumen y temperatura así presentadas pueden parecer simultáneas, estrictamente no lo son. A pesar de llevarse a cabo en un mismo ciclo del programa Arduino, hay latencias en los sensores electrónicos y en el puerto de entrada y salida de datos que impiden un registro estrictamente sincrónico. Por ser dichas latencias muy breves y mucho menores que el tiempo invertido por el motor en completar un ciclo, hemos decidido despreciar su efecto.

\section{Resultados}

Tras obtener las medidas identificamos los valores mínimos de presión y volumen, que son los que se corresponden con el inicio de un ciclo termodinámico. Esto
Tabla 2: Medidas de volumen, presión y temperatura obtenidas para un ciclo completo. Se presenta, además, el registro temporal en que se hizo la medida y el trabajo realizado por el gas en cada intervalo. No hay ningún valor apuntado para el trabajo en la primera línea, puesto que el primer intervalo es el que lleva de la primera hasta la segunda medida y no podemos determinar el trabajo hasta finalizar el intervalo.

\begin{tabular}{lcccc}
\hline $\mathbf{T}$ (h:m:s) & $\begin{array}{c}\mathbf{V} \\
(\mathbf{m l})\end{array}$ & $\begin{array}{c}\mathbf{P} \\
(\mathbf{m b a r})\end{array}$ & $\mathbf{T}\left({ }^{\circ} \mathbf{C}\right)$ & $\begin{array}{c}\mathbf{W} \\
(\mathbf{m b a r} \cdot \mathbf{m l})\end{array}$ \\
\hline 09:31:45,56 & 1,39 & 1036,65 & 31,2 & \\
09:31:45,62 & 1,39 & 1045,03 & 31,19 & 0 \\
09:31:45,64 & 1,39 & 1057,34 & 31,18 & 0 \\
09:31:45,67 & 1,39 & 1074,66 & 31,18 & 0 \\
09:31:45,71 & 0,69 & 1091,74 & 31,17 & $-758,24$ \\
09:31:45,76 & 2,1 & 1097,14 & 31,17 & 1543,16 \\
09:31:45,81 & 3,75 & 1087,05 & 31,17 & 1801,96 \\
09:31:45,86 & 3,75 & 1080,5 & 31,17 & 0 \\
09:31:45,89 & 5,17 & 1083,86 & 31,18 & 1536,70 \\
09:31:45,92 & 5,17 & 1085,17 & 31,19 & 0 \\
09:31:45,96 & 4,46 & 1080,19 & 31,18 & $-768,70$ \\
09:31:46,00 & 4,46 & 1073,69 & 31,19 & 0 \\
09:31:46,06 & 4,23 & 1057,75 & 31,19 & $-245,12$ \\
09:31:46,11 & 4,23 & 1049,97 & 31,21 & 0 \\
09:31:46,14 & 5,41 & 1043,94 & 31,2 & 1235,41 \\
09:31:46,22 & 5,17 & 1042,45 & 31,21 & $-250,37$ \\
09:31:46,27 & 5,41 & 1034,79 & 31,22 & 249,27 \\
09:31:46,32 & 4,7 & 1031,93 & 31,21 & $-733,69$ \\
09:31:46,36 & 3,52 & 1033,15 & 31,21 & $-1218,40$ \\
09:31:46,39 & 1,39 & 1032,87 & 31,2 & $-2200,31$ \\
& & & W total = 191,67 \\
\hline
\end{tabular}

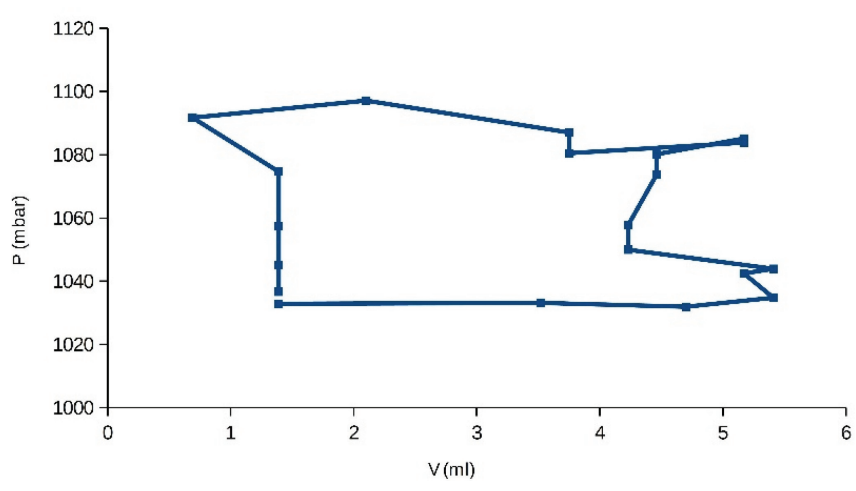

Figura 5: Diagrama presión-volumen para el ciclo termodinámico correspondiente a las medidas recogidas en la Tabla 2 .

nos permite aislar los datos correspondientes a un solo ciclo. En la Tabla 2 mostramos los resultados obtenidos para un ciclo completo. Los datos han sido editados a partir de los resultados que muestra la IDE de Arduino, a través del puerto en serie, para presentarlos en el formato adecuado. En la Figura 5 se muestra el diagrama presiónvolumen para este ciclo termodinámico del motor, representado a partir de los datos de la Tabla 2

Lo primero que observamos tanto en la tabla como en la gráfica es la irregularidad de las medidas de 


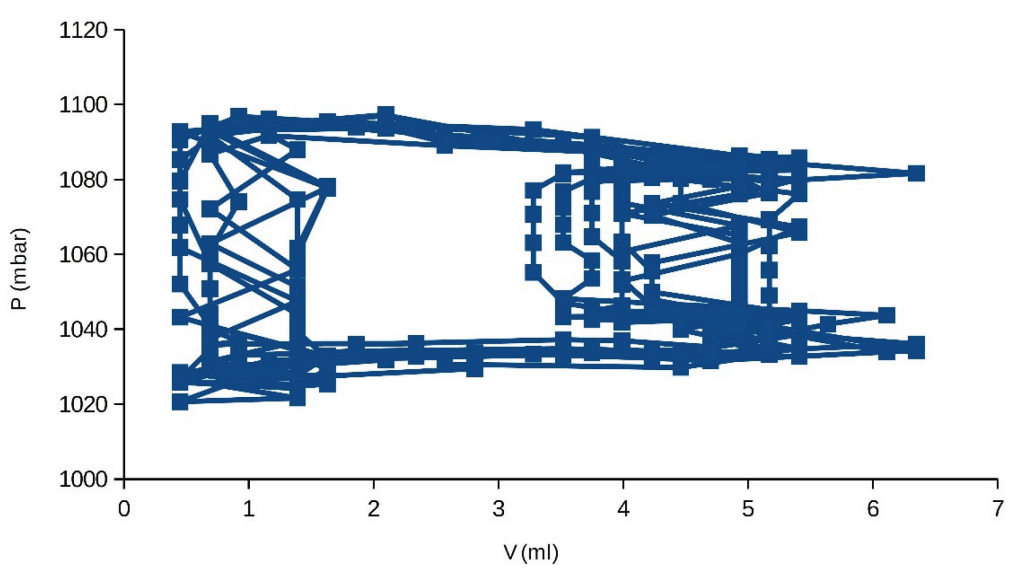

Figura 6: Diagrama termodinámico correspondiente a 10 ciclos consecutivos.

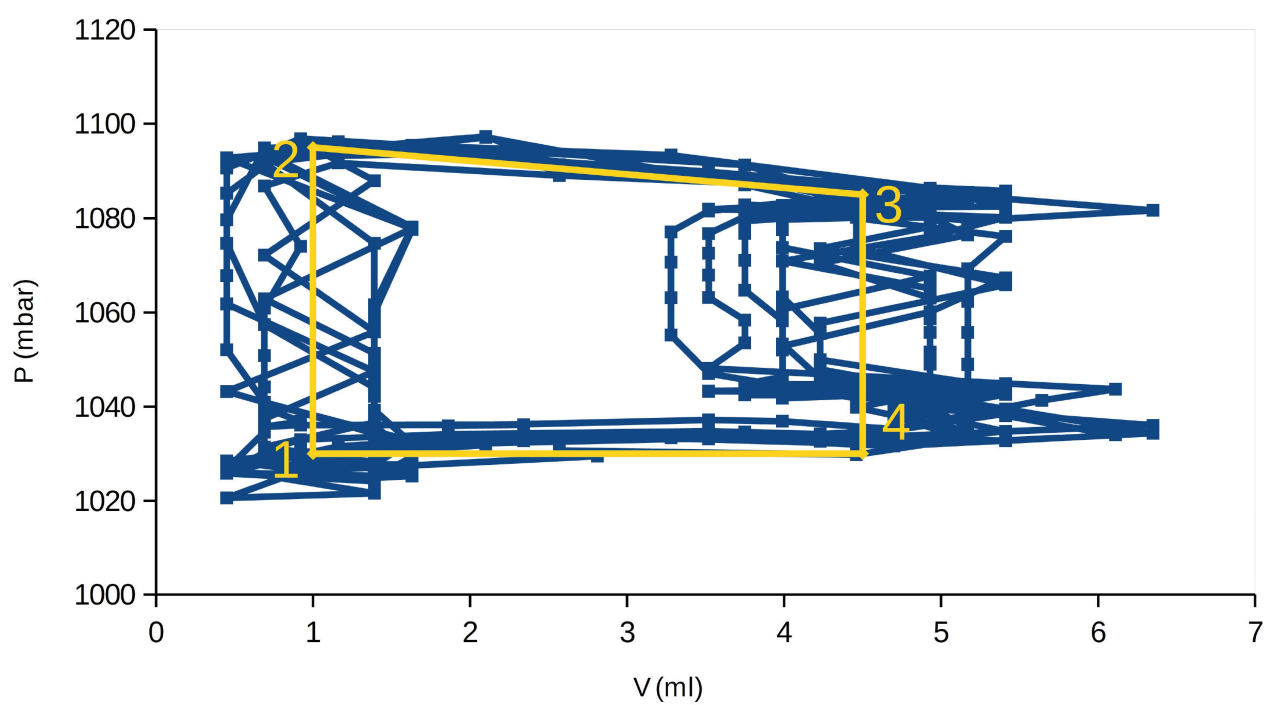

Figura 7: Estimación del ciclo termodinámico promedio (en amarillo) a partir de 10 ciclos del motor (en azul). Se han marcado los 4 puntos extremos que permiten definir las 4 etapas de las que consta el ciclo.

volumen, ya comentadas en la sección anterior. El sensor ultrasónico de posición HC-SR04, de acuerdo con las indicaciones del fabricante, tiene una sensibilidad de $3 \mathrm{~mm}$. Las fluctuaciones de $\pm 3 \mathrm{~mm}$ en la determinación de la posición de la placa metálica llevan a las fluctuaciones en la determinación del volumen que se observan tanto en la tabla como en la gráfica. Debemos señalar, además, que en ocasiones el sensor registra medidas sin sentido, correspondientes a volúmenes que no pueden ser medidos por la jeringuilla (negativos o superiores a su volumen máximo). Hemos filtrado estas medidas, eliminándolas de los registros. Para reducir el efecto de dichas fluctuaciones, hemos analizado 10 ciclos completos. En la Figura 6 se muestra el diagrama presión-volumen correspondiente a los 10 ciclos. Se puede observar que los ciclos son similares, si bien cambia su tamaño al variar de unos a otros el volumen máximo y mínimo alcanzado. Estas diferencias eran previsibles en tanto que a simple vista se observa que las oscilaciones del motor no siempre son idénticas, algunas tienen más amplitud que otras.

Sobre la gráfica hemos hecho una estimación de cuál podría ser el diagrama promedio para un ciclo. Lo hemos dibujado superpuesto en la Figura 7

\subsection{Análisis del ciclo termodinámico}

En el diagrama presión-volumen de la Figura 7 se pueden observar cuatro etapas que se corresponden con las observadas en el movimiento del motor y que ya fueron descritas en la sección 3 En la etapa 1-2 la presión aumenta mientras el volumen se mantiene (aproximadamente) constante. Cuando se alcanzan aproximadamente los 1095 mbar tiene lugar la siguiente etapa: el volumen aumenta rápidamente, la jeringuilla sube, el tubo de ensayo cambia de inclinación y el gas se desplaza al foco frío. La etapa 3-4 corresponde a una disminución de la presión producida por el enfriamiento del gas. Al 
alcanzar los 1030 mbar tiene lugar la cuarta etapa, donde el gas se comprime por la bajada de la jeringuilla. Se cierra así el ciclo termodinámico.

\subsection{Trabajo realizado por el gas}

Hemos usado los datos de la Tabla 2 y la ecuación 6 para determinar el trabajo realizado por el gas durante un ciclo. Así mismo, hemos considerado el ciclo como la suma de los intervalos que llevan al gas de cada punto del diagrama presión-volumen al siguiente punto. Como la presión no es constante a lo largo del intervalo, hemos considerado que el valor más adecuado de la presión para cada intervalo es la media aritmética del valor de la presión inicial y el valor de la presión final de dicho intervalo, por lo que la ecuación 6 anterior queda de la siguiente manera:

$$
W_{12}=\frac{p_{1}+p_{2}}{2} \cdot\left(V_{2}-V_{1}\right)
$$

Al aplicarlo al ciclo presentado en la Figura 5 hemos obtenido un trabajo de 191,7 mbar $\cdot \mathrm{ml}$, correspondiente a $1,92 \cdot 10^{-2} \mathrm{~J}$. El efecto de las fluctuaciones debidas a la sensibilidad del sensor HC-SR04 queda ahora minimizado, en la medida que los intervalos en los que el incremento de volumen haya quedado sobredimensionado son compensados por los que hayan quedado infradimensionados. Así mismo, con el fin de reducir todavía más el efecto de dichas fluctuaciones, hemos calculado el trabajo realizado por el gas durante 10 ciclos, obteniendo un valor promedio de 205,6 mbar $\cdot \mathrm{ml}$ por ciclo, correspondiente a $2,06 \cdot 10^{-2} \mathrm{~J}$. Por último, usando el diagrama promedio estimado obtenemos un valor de $210 \mathrm{mbar} \cdot \mathrm{ml}\left(2,1 \cdot 10^{-2} \mathrm{~J}\right)$ para el trabajo, similar al valor promedio calculado para los 10 ciclos.

\subsection{Calor aportado por el mechero de alcohol}

Usando una báscula hemos determinado que el mechero consume 0,73 gramos de alcohol comercial en 2 minutos. Considerando que tiene una riqueza en etanol del $96 \%$ en volumen y que la entalpía de combustión del etanol es de $1411 \mathrm{~kJ} / \mathrm{mol}$, obtenemos que el mechero aporta energía en forma de calor a un ritmo de $179 \mathrm{~J} / \mathrm{s}$.

\subsection{Calor absorbido por el gas de acuerdo con el ciclo ideal}

Para determinar el calor absorbido por el gas, primero hemos hecho una estimación de la cantidad de aire que se calienta y enfría en cada ciclo. A pesar de que el tubo de ensayo vacío tiene un volumen cercano a los $100 \mathrm{ml}$, sólo el aire en contacto con el foco caliente se va a calentar. Es más, sólo el aire desplazado por las canicas va a seguir el ciclo de calentamiento y enfriamiento. Por este motivo, hemos considerado que el volumen de aire que sigue el ciclo es:

$$
V_{\text {aire }}=2 \cdot V_{\text {canica }}
$$

Teniendo en cuenta que las canicas usadas tienen $26 \mathrm{~mm}$ de diámetro, obtenemos que el valor del volumen es de $18,4 \mathrm{ml}$.

El calor absorbido, de acuerdo con las ecuaciones correspondientes al ciclo ideal, viene determinado por la expresión:

$$
Q_{a b}=n R T_{c} \ln \frac{V_{2}}{V_{1}}+n c_{v}\left(T_{c}-T_{f}\right)
$$

Donde:

- $\mathrm{T}_{\mathrm{f}}$ es la temperatura del foco frío, de acuerdo con el sensor BMP180, $\mathrm{T}_{\mathrm{f}}=31^{\circ} \mathrm{C}$.

- $\mathrm{V}_{1}$ es el volumen inicial del gas, que hemos considerado de $18,4 \mathrm{ml}$.

- Usando la ecuación del gas ideal y aprovechando el valor de $\mathrm{P}_{1}$ indicado por el sensor $\mathrm{BMP}$, podemos calcular que $\mathrm{n}=7,6 \cdot 10^{-4} \mathrm{~mol}$.

- De acuerdo con las medidas del sensor HC-SR04, mostradas en la Figura 6, podemos estimar que el volumen de la jeringuilla varía desde $1 \mathrm{ml} \mathrm{a} \mathrm{4,5} \mathrm{ml}$. Considerando que el gas que se calienta experimenta una expansión de $3,5 \mathrm{ml}, \mathrm{V}_{2}$ toma un valor de $\mathrm{V}_{2}=21,9 \mathrm{ml}$.

- Por último, usando nuevamente la ecuación del gas ideal, y estimando que la presión antes de iniciar la etapa de compresión a volumen constante es de 1085 mbar, podemos determinar que $\mathrm{T}_{\mathrm{c}}=108^{\circ} \mathrm{C}$.

Sustituyendo todos los valores en la ecuación 10 y considerando que $\mathrm{c}_{\mathrm{v}}=20,77 \mathrm{~J} / \mathrm{mol} \cdot \mathrm{K}$, obtenemos que $\mathrm{Q}_{\mathrm{ab}}=1,63 \mathrm{~J}$. Como hemos comentado anteriormente, se corresponde con una pequeña fracción del calor aportado por el mechero.

\subsection{Rendimiento del motor}

\subsubsection{A partir del trabajo realizado por el gas y la energía aportada por el mechero}

De acuerdo con los datos, el motor invierte 0,83 segundos en completar el ciclo. Durante este tiempo, el mechero aporta 148,4 J. El rendimiento del motor es de:

$$
\varepsilon_{\text {ciclo }}=\frac{1,92 \cdot 10^{-2}}{148,4}=1,3 \cdot 10^{-4}
$$

Usando los valores promedio obtenidos a partir de 10 oscilaciones, el motor invierte 1,04 segundos en completar el ciclo medio. Durante este tiempo el mechero aporta 186,2 J. El rendimiento promedio del motor es de:

$$
\varepsilon_{\text {promedio }}=\frac{2,06 \cdot 10^{-2}}{186,2}=1,10 \cdot 10^{-4}
$$

\subsubsection{A partir del calor absorbido por el gas}

Usando el valor experimental del trabajo medio realizado por el gas en un ciclo y el valor calculado para $\mathrm{Q}_{\mathrm{ab}}$, 
podemos determinar el rendimiento del motor como:

$$
\varepsilon_{Q a b}=\frac{2,06 \cdot 10^{-2}}{1,63}=0,013
$$

Observamos que este valor del rendimiento es dos órdenes de magnitud mayor que el establecido a partir del calor aportado por el mechero. Como comentábamos en la sección 4, nuestro motor no dispone de un dispositivo específico de captación de calor y, además, este depende de la posición de la llama respecto al motor. Por tanto, este resultado era previsible en tanto que ahora sólo estamos considerando el calor absorbido por el aire y no todo el calor aportado por el mechero, que es mucho mayor.

\subsubsection{A partir del ciclo termodinámico ideal}

La ecuación del ciclo de Stirling ideal nos permite obtener otra medida del rendimiento del motor, de la siguiente manera:

$$
\varepsilon_{i d e a l}=\frac{R\left(T_{c}-T_{f}\right) \ln \frac{V_{2}}{V_{1}}}{R T_{c} \ln \frac{V_{2}}{V_{1}}+c_{v}\left(T_{c}-T_{f}\right)}
$$

Sustituyendo los valores de las correspondientes variables, que ya hemos comentado como se han medido, obtenemos que:

$$
\varepsilon_{\text {ideal }}=0,052
$$

Este resultado es cinco veces mayor que el determinado por el procedimiento anterior, lo que pone de manifiesto que nuestro motor está lejos del rendimiento de un motor de Stirling ideal. Nuestro procedimiento experimental ha requerido algunas simplificaciones que pueden justificar la obtención de un rendimiento menor que el teórico. La deducción de $\mathrm{T}_{\mathrm{f}}$ y $\mathrm{T}_{\mathrm{c}}$ requiere que las medidas de $\mathrm{P}$ y $\mathrm{V}$ sean sincrónicas, aspecto que hemos considerado como aproximación. Así mismo, considerar el ciclo experimental promedio lleva a tomar como resultado un ciclo de Stirling "suavizado", con un área menor que el ciclo teórico, como comentamos más adelante.

\subsubsection{Rendimiento de un motor con ciclo de Carnot operando entre las mismas tem- peraturas}

Por último, podemos determinar cuál sería el rendimiento teórico máximo de un motor operando a las mismas temperaturas que opera el nuestro. Para ello, determinamos el rendimiento de un motor que sigue el ciclo ideal de Carnot:

$$
\varepsilon_{\text {Carnot }}=1-\frac{T_{f}}{T_{c}}
$$

Considerando que nuestro motor opera entre $115^{\circ} \mathrm{C}$ y $31^{\circ} \mathrm{C}$, obtenemos que:

$$
\varepsilon_{\text {Carnot }}=1-\frac{304}{381}=0,20
$$

\subsubsection{Análisis de los resultados obtenidos}

Hay que destacar que los resultados obtenidos dependen de la validez de las estimaciones que se han ido exponiendo. Sin embargo, a pesar de la posible inexactitud de las medidas, los resultados obtenidos son previsibles y lógicos desde el punto de vista físico. Se observa claramente que:

$$
\varepsilon_{\text {ciclo }}<\varepsilon_{Q a b}<\varepsilon_{\text {ideal }}<\varepsilon_{\text {Carnot }}
$$

Al medir $\varepsilon_{\text {ciclo }}$ comparamos el trabajo hecho por el gas con toda la energía aportada por el mechero. Nuestro motor no tiene ningún elemento que permita al tubo de ensayo captar energía procedente de la llama de una manera eficiente, por lo que cabía esperar que el rendimiento calculado mediante este procedimiento fuese bajo. Si pudiésemos medir el rendimiento del motor como la relación entre el trabajo realizado por el mismo (elevando un objeto de masa conocida, por ejemplo) y el calor aportado por el mechero, el resultado que hubiésemos obtenido sería un rendimiento todavía menor, en tanto que parte del trabajo realizado por el gas se hubiese perdido en rozamientos y otros aspectos relacionados con el montaje de la propia máquina.

Al medir $\varepsilon_{\text {Qab }}$ sólo tenemos en cuenta el calor absorbido por el gas, por lo que el valor del rendimiento mejora enormemente. Este resultado nos permite apreciar que, con un sistema de captación de calor adecuado, el aprovechamiento energético de nuestro motor tiene un amplio margen de mejora.

Ambos valores están lejos del rendimiento del motor de Stirling ideal. El diagrama P-V de la Figura 8 muestra que el ciclo de nuestro motor difiere bastante del ciclo ideal, siendo significativamente más pequeño. Para la determinación del ciclo ideal que correspondería a nuestro motor, hemos tomado como referencia las temperaturas de los focos frío y caliente, que han sido identificados como las temperaturas de los puntos 1 y 3 en el diagrama de la Figura 7. De esta manera, hemos obtenido a un diagrama del ciclo ideal que circunscribe al diagrama experimental de nuestro motor. Observamos que nuestro motor no alcanza las presiones extremas que prevé el ciclo ideal, ya que no dispone de ningún elemento que le permita mantener el volumen una vez superada la presión mínima que lo acciona. Todo ello redunda en una "suavización" del ciclo, comentada también en otras investigaciones [14, 17.

Hemos comprobado que un motor de Carnot (ideal) que trabaje entre las mismas temperaturas que nuestro motor presenta un rendimiento 4 veces mayor. Este resultado (ecuación 17) no sólo evidencia cuán lejos estamos de construir un motor ideal, sino que también pone de relieve el bajo rendimiento de los motores térmicos reales: ningún motor térmico operando entre dichas temperaturas podrá convertir en trabajo más de la quinta parte de la energía que se le aporta como calor. Esto es un duro golpe para este tipo de tecnología, 


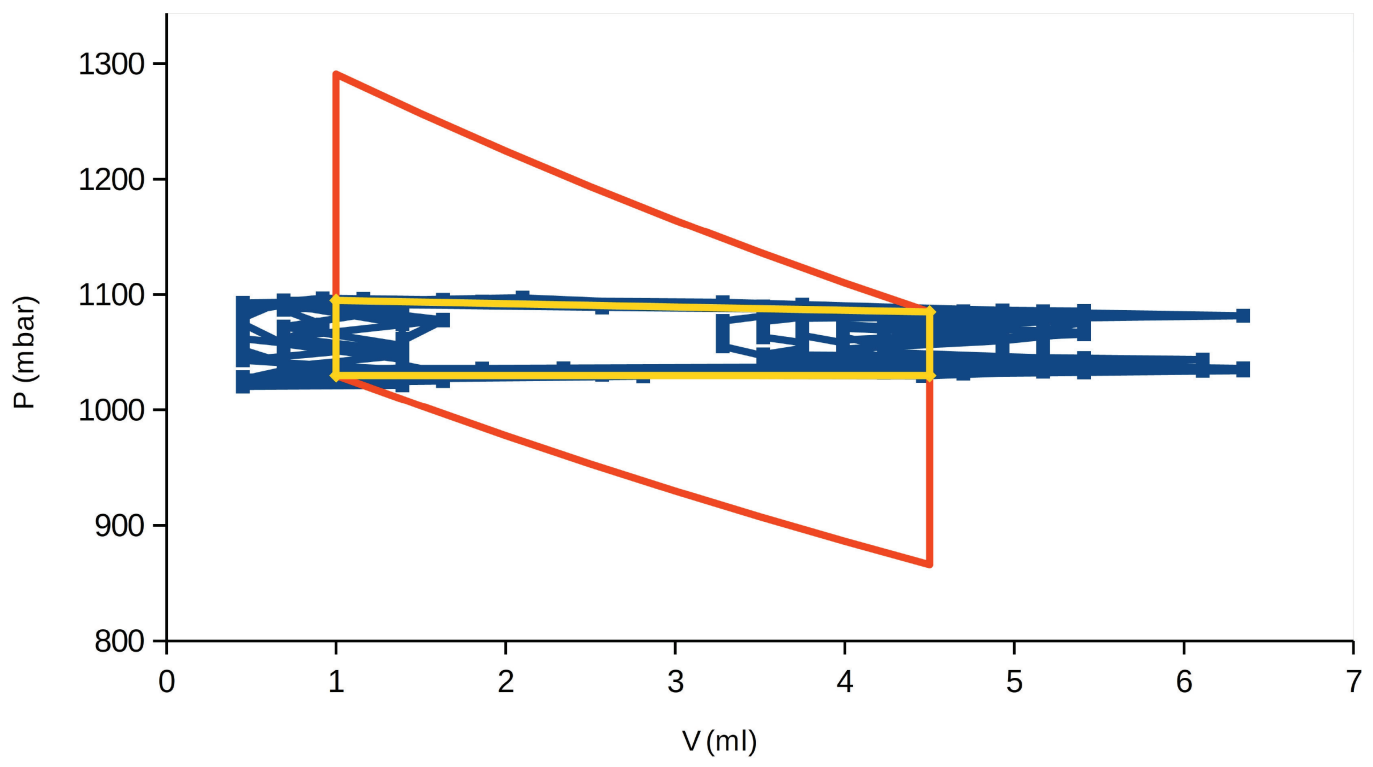

Figura 8: Se muestra en azul el diagrama experimental obtenido a partir de 10 ciclos de nuestro motor de Stirling. En amarillo se superpone el diagrama promedio estimado. En naranja se presenta el diagrama del motor de Stirling ideal que trabaja entre las mismas temperaturas que el nuestro (determinadas a partir del diagrama promedio).

especialmente por la preocupación global actual sobre el cambio climático.

Por último, hemos hecho algunas pruebas introduciendo modificaciones en el diseño del motor y analizando el impacto sobre su rendimiento. Nos ha llamado especialmente la atención que un aumento en el número de canicas contenidas en el tubo de ensayo mejora el rendimiento. Esto no se debe a que el aire contenido en el motor haga más trabajo. De hecho, el diagrama que se obtiene es más pequeño, por lo que el trabajo realizado por el gas durante un ciclo es menor. Ocurre esto porque el movimiento del motor es más rápido y el calor absorbido en cada ciclo es menor. Como consecuencia, se obtiene un rendimiento ligeramente mayor.

\section{Conclusiones}

El trabajo que hemos presentado nos ha permitido trabajar diversos aspectos referentes a los motores térmicos. A partir del estudio del ciclo termodinámico de un motor térmico, hemos constatado que podíamos establecer diversas estrategias para medir su rendimiento, valorando las dificultades y limitaciones que implicaba la puesta en práctica de cada una de ellas. Además, hemos constatado que los modelos teóricos de ciclo termodinámico que habitualmente se presentan en los textos de Física y en la enseñanza habitual distan de los que se obtienen experimentalmente al estudiar el funcionamiento de motores reales. Los procesos termodinámicos implicados (isotermos e isócoros, en el caso del motor de Stirling) son aproximaciones teóricas que solo se dan de manera aproximada a nivel experimental. Con todo, el rendimiento previsto para un motor a partir de su ciclo teórico es siempre mayor que el rendimiento medido experimentalmente. En conjunto, llama especialmente la atención (y así ha ocurrido también con los estudiantes) el bajo rendimiento que tiene la tecnología del motor térmico.

Así mismo, este trabajo nos ha permitido abordar diversos aspectos referentes a los motores térmicos con especial relevancia científico-técnica y social. Por un parte, hemos aprovechado el motor de Stirling para contextualizar los conceptos de calor y trabajo y el primer principio de la termodinámica, superando las aproximaciones teóricas y transmisivas que suelen caracterizar al tratamiento de estos conceptos en la enseñanza habitual. Por otra parte, hemos desarrollado una propuesta didáctica que permite a los alumnos trabajar habilidades relacionadas con diferentes disciplinas STEM.

En estos momentos estamos trabajando en la evaluación del impacto que tiene la aplicación de esta propuesta didáctica sobre el aprendizaje y actitudes del alumnado. Esperamos dar cuenta de estos resultados en futuras publicaciones.

\section{Anexo}

Código del programa usado para el registro de medidas con los sensores y la placa Arduino UNO

//Bloque destinado a la declaración de variables y bibliotecas

\#include < SFE_BMP180.h> //biblioteca para el sensor de presión

\#include $<$ Wire.h $>/ /$ biblioteca para el sensor de presión

\#define echoPin $11 / /$ pin de eco 
\#define trigPin $12 / /$ pasador de activación \#define button 3 //pin del botón

SFE_BMP180 bmp180;

long duration, distance; //variables usadas para calcular la distancia

float volumen;

int $\mathrm{val}=0 ; / /$ variable para almacenar el valor del botón

//Bloque destinado a la inicialización de los sensores void $\operatorname{setup}()$

\{

Serial.begin (9600);

pinMode(trigPin, OUTPUT); // Trigger y eco del

sensor de distancia

pinMode(echoPin, INPUT);

pinMode (button, INPUT); // Botón

if (bmp180.begin ()$)$

\{

Serial.println("BMP180 iniciado correctamente");

Serial.println("volumen (ml); Temperatura (C);

Presión (mbar)");

$$
\text { \} }
$$

else

\{

Serial.println("Error al iniciar el BMP180");

while(1); // bucle infinito. Si no funciona el sensor no permite medir.

\} \}

//Bloque destinado al proceso de medida void $\operatorname{loop}()$

\{

// Instrucción destinada a controlar el flujo de medidas. Sólo cuando el botón está apretado se realizan medidas.

val=digitalRead(button);

if $(\mathrm{val}==\mathrm{LOW}) / /$ Lee el valor del puerto del botón e inicia un condicional. Si está apretado hace las medidas.

\{

char status; //variables para el sensor de presión double T,P; //variables para el sensor de presión

// Bloque destinado a la medida de la distancia de la placa metálica y el volumen de la jeringuilla

digitalWrite(trigPin, LOW);

delayMicroseconds $(2)$;

digitalWrite(trigPin, HIGH);

delayMicroseconds (10);

digitalWrite(trigPin, LOW);

duration $=$ pulseIn $($ echoPin, HIGH $)$;

distance $=$ duration $* 0.17 ; / /$ Velocidad del sonido $=0,17 \mathrm{~mm} /$ microsegundo

volumen $=0.236^{*}$ distance $-24.803 ; / /$ Volumen de

la jeringuilla. Se usa la recta de calibración.

// Fin del bloque destinado a la medida del volumen
// Bloque destinado a la lectura de la presión y la temperatura del foco frío

status $=$ bmp180.startTemperature();//Inicio de lectura de temperatura

if (status $!=0$ )

\{

delay(status); //Pausa para que finalice la lectura

status $=$ bmp180.getTemperature $(\mathrm{T}) ; / /$ Obtener la temperatura

if (status $!=0$ )

\{

status $=$ bmp180.startPressure(3); //Inicio

lectura de presión

if (status $!=0)$

\{

delay(status);//Pausa para que finalice la lectura

status $=$ bmp180.getPressure $(\mathrm{P}, \mathrm{T}) ; / /$

Obtenemos la presión

// Fin del bloque destinado a la medida de la presión y la temperatura del foco frío

// Bloque destinado a transmitir las medidas al ordenador

if (status $!=0)$

\{

Serial.print("“; ");

Serial.print(volumen,2);

Serial.print(";");

Serial.print $(\mathrm{T}, 2)$;

Serial.print(";");

Serial.println $(\mathrm{P}, 2)$;

\}

\}

\}

// Fin del bloque destinado a la transmisión de medidas

\}

\section{Referencias}

[1] G. Organtini, Journal of Physics: Conf. Series 1076, 012026 (2019).

[2] J. Kiuchin, Physics Education 53, 063007 (2018).

[3] A.A. Moya, The Physics Teacher 57, 621 (2019).

[4] H.A. Wijaya y M. Khairudin, Journal of Physics: Conf. Series 1413, 012015 (2019).

[5] A.M.B. Goncalves, W.P.S. Freitas, D.D. Reis, C.R. Cena, D.C.B. Alves y D.F. Bozano. The Physics Teacher 57, 640 (2019)

[6] R. Puantha, Physics Education 54, 015009 (2019).

[7] I.N. de Oliveira, L.B. Calheiro, D.F. Bozano, N.C.G. Errobidart, M.I.A. Jardim, D.D. Reis y A. M.B. Goncalves. Revista Brasileira de Ensino de Física 42, e20190105 (2020).

[8] A.A. Moya, Physics Education 53, 055020 (2018). 
[9] P. Vale Alves, L.H. Souza Reis, C.A.S. Querino, M.A. Lima Moura, A.A. Feitosa Junior y P.A. da Silva Martins. Revista Brasileira de Ensino de Física 42, e20190304 (2020).

[10] K. Zachariadou, K. Yiasemides y N. Trougkakos, European Journal of Physics 33, 1599 (2012).

[11] J.M. Cardoso y M. Zannin, Revista Brasileira de Ensino de Física 41, e20190028 (2019).

[12] F.S. Oliveira, L.B. Calheiro, D.F. Bozano, N.C.G. Errobidart, M.I.A. Jardim, D.D. Reis y A.M. B. Goncalves. Physics Education 56, 065012 (2019).

[13] A.S. Cid y T. Correa, Revista Brasileira de Ensino de Física 41, e20180333 (2019).

[14] R.D. Spence y C.L. Foiles, The Physics Teacher 20, 38 (1982).

[15] M.D. Kutzner y M. Plantak, The Physics Teacher 52, 418 (2014)

[16] D. Clucas y S. Gutschmidt, em 3rd Annual International Conference on Industrial, Systems and Design Engineering (Atenas, 2015).

[17] Y. J. Lu, H. Nakahara y J. S. Bobowski, The Physics Teacher 58, 18 (2020).

[18] L.V. Rezende Dias y A.C. Baleeiro Alves, em Simposio Brasileiro de Sistemas Electricos (Rio de Janeiro, 2018).

[19] DOCV, Decreto $87 / 2015$, de 5 de junio, del Consell, por el que se establece el currículo y desarrolla la ordenación general de la Educación Secundaria Obligatoria y el Bachillerato en la Comunitat Valenciana, 2015. Disponible: https://www.dogv.gva.es/port al/ficha_disposicion_pc.jsp?sig $=005254 / 2015 \& \mathrm{~L}=1$

[20] M. Boada, Investigación y Ciencia 489, 84 (2017). 